BEST PRACTICE

\title{
HIV viral suppression in the era of antiretroviral therapy
}

\author{
H K Thaker, M H Snow
}

Postgrad Med J 2003;79:36-42

Altogether 42 million people worldwide have been infected with HIV, and 12 million have died over the last 20 years. Effective antiretroviral therapy has lead to sustained HIV viral suppression and immunological recovery in patients who have been infected with the virus. The incidence of AIDS has declined in the Western world with the introduction of effective antiretroviral therapy. Questions on When to start treatment?, What to start with?, How to monitor patients?, remain heavily debated. Adherence to antiretroviral treatment remains the cornerstone of effective treatment, and failure to adhere is the strongest predictor of virological failure. Long term therapy can lead to metabolic complications. Resource poor countries are dealing with difficult issues such as mother to child prevention of HIV transmission. Other treatment options are now available, with the recent introduction of fusion inhibitors, second generation non-nucleoside reverse transcriptase inhibitors, and nucleotide reverse transcriptase inhibitors to clinical practice.

See end of article for authors' affiliations

Correspondence to: Dr H K Thaker, Department of Infectious Diseases, Castle Hill Hospital, Castle Road, Cottingham, East Yorkshire HU16 5JQ, UK; hiten.thaker@hey.nhs.uk

Submitted

18 June 2002

Accepted

1 October 2002 nitially recognised in 1981, AIDS has had a devastating impact, particularly on people in the developing world. It is estimated that 42 million people worldwide, of whom two thirds live in Africa, have been infected with HIV. Twelve million people, of whom 3 million were children, have died. ${ }^{12}$

In most of the world access to effective antiretroviral therapy is prevented by economic and political shortcomings. In the developed world although effective antiretroviral therapy is generally available and the knowledge of the viral genome, life cycle, resistance mechanisms, and virus-host interaction have become increasingly sophisticated, major questions remain unanswered or unclear. Some of these questions which the following paper will examine are, When to start therapy? What to start with? Long term prospect of response and toxicity? Factors influencing treatment success or failure-that is, resistance or adherence? Other important issues that will be analysed include the impact of antiretroviral therapy on transmission of HIV infection from mother to child, strategies to deal with treatment failure, and adjunctive immunomodulatory therapy such as interleukin 2 .

It has been tempting to apply intuitive but theoretical approaches to HIV therapy (hit early!, hit hard!) but these have not necessarily been successful and may have exposed individuals to unnecessarily long antiretroviral therapy with subsequent problems of metabolic and other side effects and the appearance of and selection of resistant viral mutations. The authors are convinced that until antiretroviral drugs that are less likely to allow the development of resistance, have much better side effect profiles, and have much more "forgiving" pharmacokinetics are developed the conservative approach contained within the latest British HIV Association guidelines provide the pragmatic and safer line of treatment. We also feel that whatever the approach indicated by the virological and immunological measurements, the overriding issue is the commitment and the quality of education and support the patient has. Patients who are, for whatever reason, seriously non-adherent to therapy may exhaust all available treatment options over relatively short periods of time. Treatment should be deferred until the time and circumstances are right. No HIV treatment programme can be effective without patient confidence and knowledge supported by ongoing adherence education and support.

\section{ANTIRETROVIRAL DRUGS}

There are 16 licensed drugs available in the UK at present (table 1). Multidrug "highly active antiretroviral therapy" (HAART) reduces the incidence of opportunistic infections and slows the progression from HIV to AIDS and from AIDS to death (fig 1). ${ }^{3}$ Combining agents produces more complete viral suppression, which in turn limits the emergence of drug resistance, and provides more effective antiretroviral treatment even when mixtures of drug resistant and drug sensitive strains are present. The standard recommendation is for triple combination therapy. These combinations can be triple nucleoside reverse transcriptase inhibitor (NRTI) regimens, nonnucleoside reverse transcriptase inhibitor (NNRTI) based regimens (2NRTI and INNRTI), and protease inhibitor (PI) based regimens (table 2 ). The goal of a class sparing regimen, such as triple NRTI therapy, is to preserve or "spare" one or more classes of drugs for later use. Sequencing drugs in this fashion, might extend the overall long term effectiveness of the available treatment options for an individual, but cross resistance between certain drugs in the same class needs to be taken into account. Moreover, this strategy makes it possible to selectively avoid certain side effects associated with a particular class of drugs. The efficacy of protease inhibitor-containing

Abbreviations: HAART, highly active antiretroviral therapy; NNRTI, non-nucleoside reverse transcriptase inhibitor; NRTI, nucleoside reverse transcriptase inhibitor; $\mathrm{PI}$, protease inhibitor 
Table 1 Current available antiretroviral drugs

\begin{tabular}{lll}
\hline NRTI & NNRTI & PI \\
\hline Thymidine & - Nevirapine (NVP) & - Ritonavir \\
- Zidovudine (AZT) & - Efavirenz (EFV) & - Saquinavir \\
- Stavudine (d4T) & - Delavirdine (DLV) & - Nelfinavir \\
Non-thymidine & & - Amprenavir \\
- Didanosine (ddl) & & Boosted PI \\
- Zalcitabine (ddC) & & \\
- Lamivudine (3TC) & & \\
- Abacavir (ABC) & & \\
Combination & & \\
- Combivir (AZT/3TC) & & \\
- Trizivir (AZT/3TC/ABC) & & \\
\hline
\end{tabular}

Table 2 Sequential pathways in treatment

\begin{tabular}{llll}
\hline $2 \mathrm{NRTI}+\mathrm{PI}$ & $2 \mathrm{NRTI}+\mathrm{NNRTI}$ & $3 \mathrm{NRTI}$ & $\mathrm{NRTI}+\mathrm{NNRTI}+\mathrm{PI}$ \\
$\downarrow$ & $\downarrow$ & $\downarrow$ & $\downarrow$ \\
$2 \mathrm{NRTI}+$ ritonavir + PI & $2 \mathrm{NRTI}+\mathrm{PI}$ & $2 \mathrm{NRTI}+\mathrm{NNRTI}$ & $2 \mathrm{NRTI}+2 \mathrm{PI}$ \\
$\downarrow$ & $\downarrow$ & $\downarrow$ & $\downarrow$ \\
$2 \mathrm{NRTI}+\mathrm{NNRTI}$ & $2 \mathrm{NRTI}+2 \mathrm{PI}$ & $2 \mathrm{NRTI}+\mathrm{PI}$ & Mega-HAART \\
$\downarrow$ & $\downarrow$ & $\downarrow$ & \\
Mega-HAART & Mega-HAART & Mega-HAART & \\
\hline
\end{tabular}

HAART regimens includes durable viral load suppression, partial immunological restoration, and decreased incidence of AIDS and death. ${ }^{45}$ Initially protease inhibitor regimens were felt to have a potency advantage, but viral load suppression and $\mathrm{CD} 4+\mathrm{T}$ cell responses of a similar scale have been achieved with selected protease inhibitor-sparing regimens. However, it is not yet known whether these will provide comparable efficacy in clinical endpoints and there are concerns that triple NRTI regimens may not be ideal therapy for patients with very high viral loads.

\section{GOALS OF THERAPY}

The current goal of effective antiretroviral therapy is to maintain health and wellbeing with minimum drug side effects for as long as possible. This is usually achieved by suppressing the viral load to below the limit of detection of the most sensitive assay for as long as possible. This usually leads to preservation or restoration of immunological function (box 1$)^{6}$ but discord-

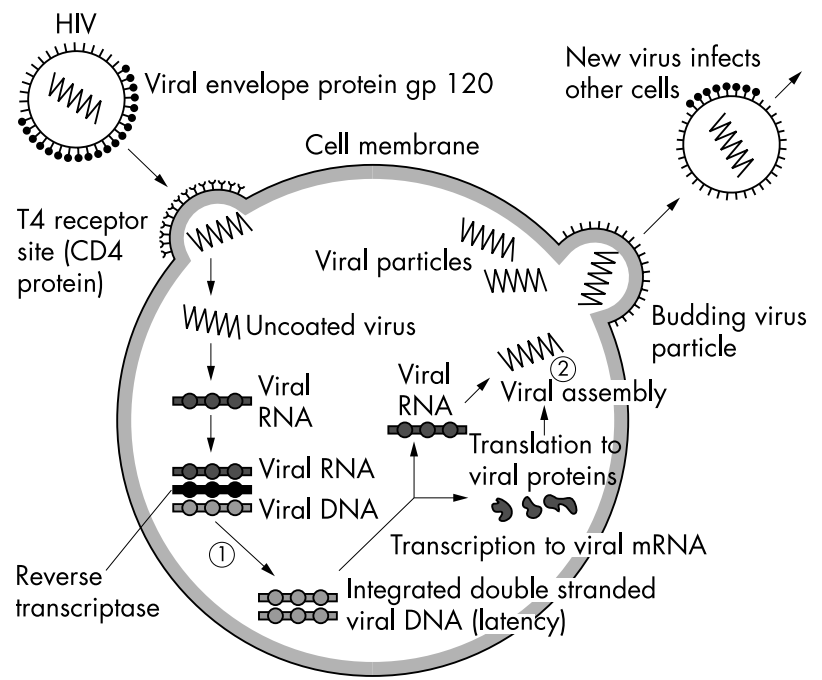

Figure 1 Life cycle of HIV virus. (1) Reverse transcriptase enzyme; (2) protease enzyme. ance may occur. Eradication of HIV infection ${ }^{7}$ cannot be achieved with currently available antiretroviral regimens due to the establishment of a pool of latently infected CD4 + T cells during the very earliest stages of acute HIV infection that persist with an extremely long half life, even with prolonged suppression of plasma viraemia to $<50$ copies $/ \mathrm{ml} .{ }^{68}$ Suppression of viral load, restoration, and/or preservation of immunological function leads to improvement of quality of life, and reduction of HIV related morbidity and mortality. ${ }^{911}$ This has been illustrated by the reduction in HIV death rates in both the UK and USA after the introduction of triple therapy (figs 2 and 3 ).

Plasma viraemia is a strong prognostic indicator in HIV infection, ${ }^{12}$ and persistent reduction in plasma viraemia achieved with antiretroviral therapy may account for some of the clinical benefit associated with therapy. ${ }^{13}$ However the degree of suppression of the CD4 count indicates the predisposition to opportunistic infections and hence shorter term risks of morbidity and progression to AIDS. A sustained rise in CD4+ T cell counts and partial immune restoration is very important. HAART often leads to increases in the CD4+ T cell count of 100-200 cells/ml or more, although individual

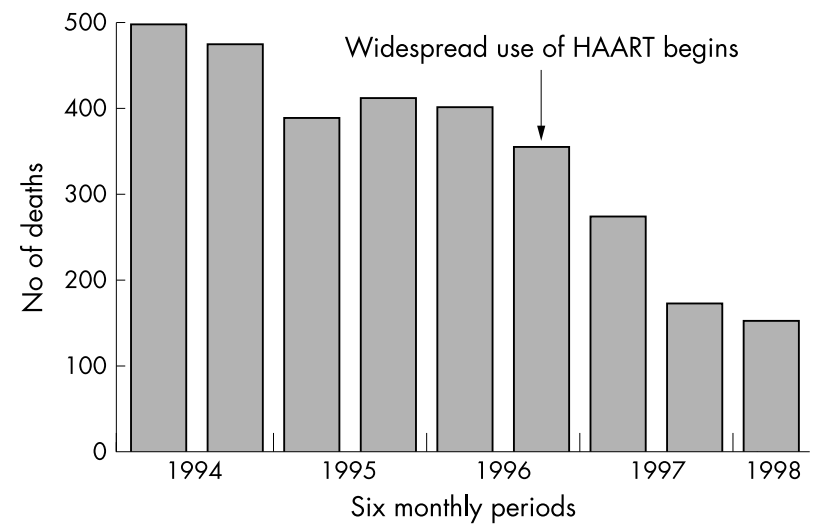

Figure 2 Deaths of HIV infected adults in the same six month periods over five years. 


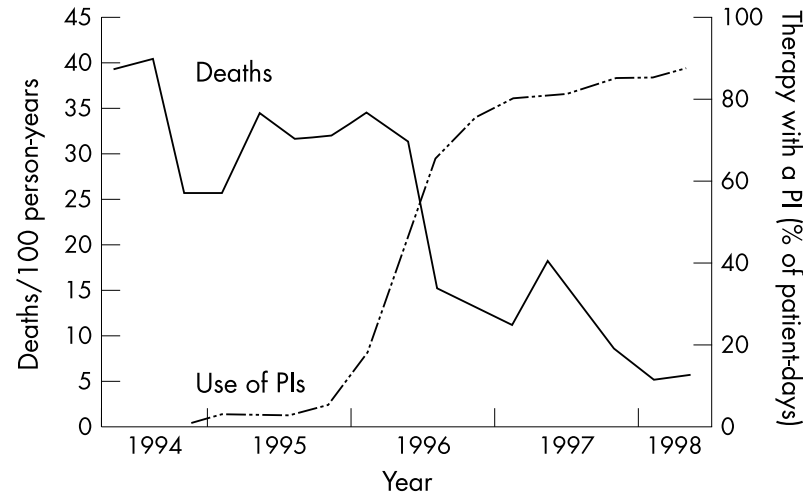

Figure 3 Mortality and protease inhibitor use; Pl, protease inhibitor.

\section{Box 1: Goals of treatment and tools to achieve them}

\section{Goals}

- Maximal and durable suppression of viral load.

- Restoration and/or preservation of immunological function.

- Improvement of quality of life.

- Reduction in HIV related morbidity and mortality.

Tools

- Maximise adherence to the antiretroviral regimen.

- Sequence drugs rationally.

- Preserve future treatment options.

- Use resistance testing in selected clinical settings.

responses are quite variable. CD4+ T cell responses are generally related to the degree of viral load suppression but also are influenced in magnitude by the nadir CD4 count. ${ }^{14}$ In turn, continued viral load suppression is more likely among those who achieve higher CD4+ T cell counts during therapy. ${ }^{15}$ Predictors of virological success include low baseline viraemia and high baseline CD4+ $\mathrm{T}$ cell count, ${ }^{16}$ rapid decline of viraemia, ${ }^{17}$ decline of viraemia to $<50$ HIV RNA copies $/ \mathrm{ml}^{17}$ adequate serum levels of antiretroviral drugs, ${ }^{17}{ }^{18}$ and better than $95 \%$ adherence to the drug regimen. ${ }^{19}$

\section{CURRENT CONTROVERSIES}

There are questions that have yet to be fully answered. Most importantly are those regarding the timing of the initiation of treatment, the durability of viral suppression and clinical response, and the optimal "salvage" regimens for patients failing therapy either clinically or virologically. ${ }^{3}$

\section{When to start treatment}

The answer to this question remains unclear. Although, now it is thought favourable to start treatment later rather than earlier, the decision should still be on an individual risk/benefit basis (table 3). Eradication with early therapy is not a possibility but at the other extreme treatment outcome is definitely worse when disease is advanced, with low CD4 counts. Cumulative toxicities develop related to duration of
Early Late

\begin{tabular}{ll}
\hline Benefits & Benefits \\
\hline Progression unlikely & Reduced toxicity \\
Reduced viral diversity & Preserve future options \\
Delay or prevent immune compromise & Delay resistance \\
Decreased risk of HIV transmission & Better new drugs developed \\
Risks & Risks \\
Increased toxicity & Increased viral diversity \\
Treatment fatigue & Increased risk of transmission \\
Drug related reduced quality of life & Progression likely \\
Limitation of future options & $\begin{array}{l}\text { Opportunistic infection } \\
\text { more likely }\end{array}$
\end{tabular}

Figure 4 Risk/benefit analysis of early versus later therapy.

therapy with some agents and maintaining adherence becomes a burden to both the clinician and the patient. On the other hand cohort studies have shown that early initiation of therapy has dramatically reduced death rates and as disease progresses the effect of standard antiretroviral treatment may in fact diminish. The risk/benefit analysis of early versus later therapy is shown in fig 4 .

\section{What to start with}

The choice of drugs to achieve success in the initial regimen must be balanced against the need to preserve future treatment options in case of failure. There are a number factors that may influence the initial choice of antiretrovirals. Potency of viral suppression is not the only factor. Adherence is vital and this depends on easy to take regimens with low pill burden and limited toxicity. Knowledge of resistance mutations selected by these initial regimens will have a bearing on subsequent treatment.

\section{Monitoring patients}

Viral loads measured at weeks 4 and 8 are useful predictors of viral suppression by week 24 and should be monitored ${ }^{20}$ For previously antiretroviral-naive patients whose viral loads are still above 10000 , after eight weeks of HAART, there is an urgent need to assess adherence to therapy, drug levels and viral resistance, so management can be modified accordingly to reduce the rate of later virological failure. ${ }^{20}$ Current standard of care requires a careful monitoring of the full blood count, biochemical profile, lipid levels, lactate, and blood glucose to detect drug side effects. Patients should be warned of general and drug specific side effects in pre-therapy education.

\section{Adherence}

Good adherence increases the likelihood of sustained virological control, which is important for reducing HIV related morbidity and mortality. Conversely, poor adherence by allowing periods of viral replication leads to the development of drug resistance, limiting the effectiveness of treatment. ${ }^{21}$ When HAART is started in a routine clinic setting viral suppression is achieved substantially less frequently than in patients in controlled clinical trials. In several studies, non-adherence to

Table 3 When to start treatment in asymptomatic patients

\begin{tabular}{ll}
\hline Surrogate markers & Recommendation \\
\hline CD4 count $>350$ cells/ $\mu$ land any viral load & $\begin{array}{l}\text { Defer treatment } \\
\text { Start treatment within this range, taking into account the rate } \\
\text { CD4 count } 200-350 \text { cells/ } \mu \text { l }\end{array}$ \\
of CD4 decline, symptoms, patient's wishes, and viral load \\
Treat
\end{tabular}


HAART was the strongest predictor of failure to achieve viral suppression below the level of detection. ${ }^{21} \mathrm{~A}$ high degree of adherence where $90 \%-95 \%$ of the doses must be taken is necessary for optimal virological suppression with HAART. Lesser degrees of adherence are more often associated with virological failure ${ }^{19}$ Predictors of poor adherence to HIV medications include poor clinician-patient relationship, active drug and alcohol use, active mental illness (in particular depression), lack of patient education and inability of patients to identify their medications, ${ }^{22}$ and lack of reliable access to primary medical care or medication. ${ }^{23}$ Medication side effects including metabolic and morphological side effects of HAART are also associated with poor adherence. ${ }^{24}$

Predictors of good adherence include availability of emotional and practical life supports, the ability of patients to fit the medications into their daily routine, the understanding that poor adherence leads to resistance, the recognition that taking all medication doses is important, and feeling comfortable taking medications in front of people. It may also be helpful for patients to bring their medications and a medication diary to clinic visits. ${ }^{24}$

Strategies to improve adherence can include establishing a commitment to a treatment plan. Patients must understand that the first HAART regimen has the best chance of long term success. ${ }^{25}$ Daily or weekly pillboxes, timers with alarms, pagers, and other devices may be useful. Patients should be well informed of side effects that can affect the ability to adhere to treatment. Some of the medications require food restrictions in timing and content (for example, didanosine) and others an increase in fluid intake to prevent renal calculi (for example, indinavir) and these should be identified and their acceptability confirmed before starting therapy. Temporary postponement of HAART initiation has been proposed for patients with identified risks for poor adherence. ${ }^{26}$ A trusting relationship with a multidisciplinary team is essential and there should be an ongoing monitoring of adherence by the team. There is evidence that adherence wanes over time, even in highly adherent patients due to "pill fatigue", ${ }^{26}$ and thus an adherence check and reinforcement at every clinical encounter is essential. Where possible regimens should be simplified by reducing the number of pills and the frequency of dosing, and by minimising drug interactions and side effects. Directly observed therapy has been shown to be successful in the management of HIV, specifically in patients who are poorly adherent to medications. Certain antiretrovirals such as efavirenz and didanosine have been shown to be effective once a day treatment options. However suboptimal adherence in the setting of once a day treatment can be disastrous. ${ }^{27}$

Thus failure to adhere to HAART is the strongest predictor of virological failure. ${ }^{28}$

\section{METABOLIC COMPLICATIONS OF ANTIRETROVIRAL THERAPY \\ Lipodystrophy}

Lipodystrophy, dyslipidaemia, and insulin resistance may complicate protease inhibitor containing antiretroviral therapy. Changes in body fat distribution is referred to as "lipodystrophy syndrome". This syndrome has no uniform case definition and variables are poorly understood. Clinical findings include central obesity with visceral fat accumulation, dorsocervical fat accumulation ("buffalo hump"), lipomas, peripheral fat wasting, muscular wasting with venous prominence, facial thinning, and breast enlargement. ${ }^{29}{ }^{30}$ Lipodystrophy has been associated with the use of protease inhibitors, but may occur with NRTI therapy or even in the absence of therapy. ${ }^{31}$ Compared with protease inhibitor-associated lipodystrophy, the NRTI-associated syndrome(s) may be associated with recent onset fatigue, nausea, and weight loss. Changes in triglycerides and/or cholesterol have occurred with or without the clinical findings of fat maldistribution..$^{32}$ Management of this condition is difficult, but switching from HIV protease inhibitor therapy leads to improved lipids. Various other treatment options have been tried including drugs such as statins, fibrates, thiozolidiones, and metformin. In some instances cosmetic procedures may be required.

\section{Lactic acidosis/hepatic steatosis}

Lactic acidosis and hepatomegaly with steatosis can occur during use of NRTIs and can be associated with a high fatality rate. ${ }^{33}$ Risk factors for the development of this toxicity include female gender, obesity, and prolonged use of NRTIs. ${ }^{34}$ Mitochondrial toxicity is one possible mechanism of cellular injury as NRTIs inhibit DNA polymerase, which is the enzyme responsible for mitochondrial DNA synthesis. The ensuing mitochondrial dysfunction may result in lactic acidosis and hepatic steatosis and subsequently myopathy, cardiomyopathy, pancreatitis, and peripheral neuropathy.35 Antiretroviral treatment should be suspended if clinical and laboratory manifestations of the lactic acidosis syndrome occur.

\section{Hyperglycaemia/diabetes mellitus}

Hyperglycaemia, new onset diabetes mellitus, diabetic ketoacidosis, and exacerbation of pre-existing diabetes mellitus have been reported in patients receiving HAART. ${ }^{36}$ These metabolic derangements are strongly but not exclusively associated with protease inhibitor use. ${ }^{32}$ The definite pathogenesis of these abnormalities is unknown, though insulin resistance has been implicated. There are no data to aid in the decision to continue or discontinue drug treatment. There are ongoing studies examining the potential of reversing insulin resistance after switching from protease inhibitor-containing HAART regimens to NNRTI based regimens; the results have so far been inconclusive.

\section{Rash}

Rash is a relatively common toxicity encountered during use of NNRTIs. A significant minority (occurring in up to approximately $5 \%$ of patients receiving NNRTIs) of these rashes are severe. Milder rashes may resolve on treatment. Hypersensitivity to abacavir can be life threatening and patients with this reaction should not be re-exposed to this drug.

\section{Other side effects}

Other common side effects include neuropsychological disturbances with efavirenz (especially vivid dreams), anaemia with zidovudine, and hepatitis with neverapine (particularly if patients have concomitant viral hepatitis particularly hepatitis C).

\section{VIRAL RESISTANCE}

Testing for HIV resistance to antiretroviral drugs is an important aid in guiding antiretroviral therapy. Combined with a detailed drug history and combatting the lack of adherence, these assays help to maximise the benefits of antiretroviral therapy. Many studies in treatment experienced patients have shown that the presence of drug resistance (identified by either genotyping or phenotyping resistance assays) can lead to failure of antiretroviral treatment. Genotyping assays detect drug resistance mutations that are present in the viral reverse transcriptase and protease genes. Interpretation of these assays requires a knowledge of the range of mutations conferring resistance as well as the potential for cross resistance within a drug class. Phenotyping assays measure the ability of viruses to grow in various concentrations of antiretroviral drugs. Resistance assays should be performed while the patient is taking antiretroviral drugs. Prior treatment history is very important, as without drug selection pressures significant mutations may be submerged by wild type virus only to re-emerge when inappropriate second line treatment is given. 
When resistance testing was compared to clinical judgment in guiding antiretroviral therapy, studies have shown that resistance testing is superior. ${ }^{37}{ }^{38}$ Similarly, a recent prospective, randomised, multicentre trial has shown that treatment selected on the basis of phenotypic resistance testing significantly improves the virological response to antiretroviral therapy. ${ }^{39}$ Thus, resistance testing appears to be a useful tool in selecting active drugs when changing antiretroviral regimens in the setting of virological failure. Similar rationale applies to the potential use of resistance testing in the setting of suboptimal viral load reduction. There are currently no prospective data to support the use of one type of resistance assay over the other (that is, genotyping $v$ phenotyping). Results from large studies like ERA (Evaluation of Resistance Assays) are awaited; this should identify which test would be most appropriate in what situation.

The use of resistance testing before initiation of antiretroviral therapy in chronic HIV infection could be considered or a sample may be stored for subsequent analysis if there is failure of the initial regimen. Pre-treatment resistance testing may be indicated if there is evidence of high level resistance in the community. Table 4 summarises guidance for the use of resistance assays.

\section{IMMUNE RECOVERY}

The eventual degree of immune recovery achievable with HAART remains to be established. The effects were studied for a prolonged period in 41 patients of whom $88 \%$ had plasma HIV RNA levels below the limit of detection after 96 weeks of treatment. Improved immune function, including HIV specific immunity was demonstrated. ${ }^{40}$ The rise of CD4 T cells in those with $>3 \log$ viral suppression is the best available indicator of the potential for natural CD4 regeneration in HIV infected patients. However, since still greater CD4 count rises may be seen when more potent regimens become available, the total regenerative capacity of CD4 cells may then be studied. ${ }^{13}$ Patients with prolonged undetectable plasma HIV-1 RNA levels during antiretroviral therapy do not invariably show immune restoration. Naive $T$ cell recovery in the setting of complete viral suppression is a gradual process, similar to that reported for immune recovery in adults after chemotherapy and bone marrow transplantation. ${ }^{41}$ The appearance of naive T cells $s^{42}{ }^{43}$ and evidence of residual thymic function in patients receiving $\mathrm{HAART}^{44}$ suggest that partial immune reconstitution frequently may occur in these patients. Further evidence of functional immune restoration can be found in the return during HAART of in vitro responses to microbial antigens associated with opportunistic infections, ${ }^{45}$ and the lack of cases of Pneumocystis carinii pneumonia among patients who discontinued primary $P$ carinii pneumonia prophylaxis when their CD4+ T cell counts rose to $>200$ cells $/ \mathrm{mm}^{3}$ during HAART. $^{46}$

\section{VIRAL PERSISTENCE}

HAART can suppress HIV type 1 plasma viraemia to undetectable levels for three years or more. However during treatment with HAART, low level replication continues and virus resides latently in resting memory $\mathrm{CD} 4+\mathrm{T}$ lymphocytes, creating a major obstacle to HIV-1 eradication. If treatment is discontinued, viral rebound occurs in the majority of patients. This viral rebound could originate from the latent reservoir or in patients with incomplete viral suppression it may have resulted from ongoing low level replication of HIV in lymphoid tissues and other sanctuary sites. ${ }^{47}$ Initial overoptimistic calculations involving the half lives of the infected cells (estimated to be 14-21 days) suggested that three years continuous HAART might achieve complete eradication. However, several studies have determined that the half lives of chronically infected cells are in the order of 6-44 months and new estimates indicate that 60 years of completely suppressive therapy might be needed to eradicate the virus. ${ }^{48}$ Thus, there has been movement toward combining HAART with various means of augmenting and/or reconstituting the host's immune system, especially HIV-1 specific immune responses. ${ }^{49}$

\section{MOTHER-CHILD TRANSMISSION}

The ACTG 076 trial showed that zidovudine reduces the risk of perinatal HIV transmission by two thirds. ${ }^{50}$ The treatment regimen was zidovudine started at 14 weeks' gestation in a standard dose of $300 \mathrm{mg}$ twice a day and during labour given intravenously at $2 \mathrm{mg} / \mathrm{kg}$ over one hour followed by continuous infusion of $1 \mathrm{mg} / \mathrm{kg}$ intravenously until delivery. The newborn was started on oral administration of zidovudine $2 \mathrm{mg} / \mathrm{kg}$ every six hours for the first six weeks of life beginning 8-12 hours after birth. This regimen was shown to reduce the risk of perinatal transmission by $66 \%$ from $25 \%$ to $8 \%$. Recently there have been a number of trials demonstrating the efficacy of short courses of antenatal/intrapartum zidovudine to prevent perinatal transmission. These results are important for resource poor settings and when HIV infection is diagnosed late in pregnancy or during labour. A trial in Thailand where zidovudine was given for four weeks antenatally in standard doses followed by $300 \mathrm{mg}$ every three hours orally during labour reduced perinatal transmission by $50 \%$ compared with placebo. ${ }^{51}$ In Africa it has been demonstrated that a single $200 \mathrm{mg}$ oral dose of nevirapine given to the mother at the onset of labour combined with a single $2 \mathrm{mg} / \mathrm{kg}$ oral dose

Table 4 Recommendation for the use of drug resistance assays

\begin{tabular}{ll}
\hline Clinical setting/recommendation & Rationale \\
\hline $\begin{array}{l}\text { Recommended } \\
\text { - Virological failure during HAART }\end{array}$ & $\begin{array}{l}\text { - Determine the role of resistance in drug failure and maximise the number } \\
\text { of active drugs in the new regimen if indicated } \\
\text { Determine the role of resistance and maximise the number of active drugs } \\
\text { in the new regimen if indicated } \\
\text { therapy }\end{array}$ \\
$\begin{array}{l}\text { Consider } \\
\text { - Acute HIV infection }\end{array}$ & $\begin{array}{l}\text { - Determine if drug resistant virus was transmitted and change regimen } \\
\text { accordingly }\end{array}$ \\
$\begin{array}{l}\text { Not generally recommended } \\
\text { - Chronic HIV infection before initiation of therapy }\end{array}$ & $\begin{array}{l}\text { - Uncertain prevalence of resistant virus. Current assays may not detect } \\
\text { minor drug resistant species } \\
\text { Drug resistance mutations may become minor species in the absence of } \\
\text { selective drug pressure. Current assays may not detect minor drug } \\
\text { resistant species } \\
\text { Resistance assays cannot be reliably performed because of low copy } \\
\text { number of HIV RNA }\end{array}$ \\
\hline
\end{tabular}


given to the infant at 48-27 hours of age reduced transmission by nearly $50 \% .^{52}$ If women conceive on therapy and have total viral suppression during the time of delivery the same antiretroviral regimen should be continued and the neonate prescribed zidovudine as above. If the infant was to present after delivery then post-exposure prophylaxis with either single drug zidovudine or triple combination therapy should be given to the baby for a period of four weeks. Obstetric factors effect mother-child transmission rates. A comparison of pre-labour caesarean section and vaginal delivery in HIV infected women was carried out in Europe. ${ }^{53}$ This study confirmed that elective pre-labour caesarean section reduced perinatal transmission by $70 \%$. Breast feeding increases HIV transmission rates by $14 \%$ and in the UK, where safe infant feeding alternatives are available, HIV infected women are advised not to breast feed. In the developing world if there is no access to safe water supplies this may not be a safe option.

\section{CONCLUSION}

HAART has had a significant effect on background HIV morbidity reducing the incidence of opportunistic infections ${ }^{4}$ and HIV mortality rates. ${ }^{9}$ Even monotherapy regimens significantly reduce mother-child transmission rates. However, with currently available drugs eradication is not a possibility and achieving and maintaining viral suppression requires stringent adherence to treatment. Failure to do so encourages selection of resistant viral strains which have implications for both personal and public health. Therefore adherence education, ongoing support, and treatment components and dosing to fit lifestyle are critically important issues. We need more potent, better tolerated, and more forgiving drugs to increase the duration of benefit in the developing world. In some parts of the developing world even generic drugs are out of reach for treating HIV disease, but it is here that affordable effective antiretroviral therapy regimens for preventing mother-child transmission should be a priority.

\section{Authors' affiliations}

H K Thaker, Department of Infectious Diseases, Castle Hill Hospital, Cottingham, East Yorkshire

M H Snow, Department of Infection and Tropical Medicine, University of Newcastle Medical School, Newcastle General Hospital, Newcastle upon Tyne

\section{REFERENCES}

1 Anonymous. The global HIV and AIDS epidemic, 2001. MMWR Morb Mortal Wkly Rep 2001;50(21):434-9.

2 Anonymous. HIV and AIDS-United States, 1981-2000. MMWR Morb Mortal Wkly Rep 2001;50(21):430-4

3 Horowitz HW, Telzak EE, Sepkowitz KA, et al. Human immunodeficiency virus infection, part I. Disease-A-Month 1998;44:545-606.

4 Hogg RS, Yip B, Kully C, et al. Improved survival among HIV-infected patients after initiation of triple-drug antiretroviral regimens. Can Med Assoc J 1999:160:659-65.

5 Cameron DW, Heath-Chiozzi M, Danner S, et al. Randomised placebo-controlled trial of ritonavir in advanced HIV-1 disease. The Advanced HIV Disease Ritonavir Study Group. Lancet 1998;351:543-9.

6 Finzi D, Hermankova M, Pierson T, et al. Identification of a reservoir for HIV- 1 in patients on highly active antiretroviral therapy. Science 1997:278: 1295-300.

7 Habib ATH, Snow MH, Ong ELC. Efficacy of HAART in clinical practice. 13th World AIDS Conference, 2000. Durban, 2000.

8 Wong JK, Hezareh M, Gunthard HF, et al. Recovery of replication-competent HIV despite prolonged suppression of plasma viremia. Science 1997;278:1291-5.

9 Palella FJ Jr, Delaney KM, Moorman AC, et al. Declining morbidity and mortality among patients with advanced human immunodeficiency virus infection. HIV Outpatient Study Investigators. N Engl J Med 1998;338:853-60.

10 Vittinghoff E, Scheer S, O'Malley P, et al. Combination antiretroviral therapy and recent declines in AIDS incidence and mortality. J Infect Dis $1999 ; 179: 717-20$.

11 O'Brien WA, Hartigan PM, Martin D, et al. Changes in plasma HIV-1 RNA and CD4+ lymphocyte counts and the risk of progression to AIDS Veterans Affairs Cooperative Study Group on AIDS. N Engl J Med 1996;334:426-31.
12 Mellors JW, Rinaldo CR, Gupta P, et al. Prognosis in HIV-1 infection predicted by the quantity of virus in plasma. Science 1996;272:116770 .

13 Staszewski S, Miller V, Sabin C, et al. Determinants of sustainable CD4 lymphocyte count increases in response to antiretroviral therapy. Aids 1999;13:951-6.

14 Miller V, Staszewski S, Sabin C, et al. CD4 lymphocyte count as a predictor of the duration of highly active antiretroviral therapy-induced suppression of human immunodeficiency virus load. J Infect Dis 1999; 180:530-3.

15 Kaufmann D Pantaleo G, Sudre $P$, et al. CD4-cell count in HIV-1-infected individuals remaining viraemic with highly active antiretroviral therapy (HAART). Swiss HIV Cohort Study. Lancet 1998;351:723-4

16 Deeks SG, Hecht FM, Swanson M, et al. HIV RNA and CD4 cell count response to protease inhibitor therapy in an urban AIDS clinic: response to both initial and salvage therapy. AIDS 1999;13:F35-43.

17 Powderly WG, Saag MS, Chapman S, et al. Predictors of optimal virological response to potent antiretroviral therapy. AIDS virological response

18 Montaner JS, Reiss P, Cooper D, et al. A randomized, double-blind trial comparing combinations of nevirapine, didanosine, and zidovudine for HIV-infected patients: the INCAS Trial. Italy, The Netherlands, Canada and Australia Study. JAMA 1998;279:930-7.

19 Webber MP, Schoenbaum EE, Gourevitch MN, et al. A prospective study of HIV disease progression in female and male drug users. AIDS 1999:13:257-62.

20 Lepri AC, Miller V, Phillips AN, et al. The virological response to highly active antiretroviral therapy over the first 24 weeks of therapy according to the pre-therapy viral load and the weeks $4-8$ viral load. AIDS 2001;15:47-54.

21 Paterson DL, Swindells S, Mohr J, et al. Adherence to protease inhibitor therapy and outcomes in patients with HIV infection. Ann Intern Med $2000 ; 133: 21-30$

22 Chesney MA. Factors affecting adherence to antiretroviral therapy. Clin Infect Dis 2000;30(suppl 2):S171-6.

23 Shapiro MF, Morton SC, McCaffrey DF, et al. Variations in the care of HIV-infected adults in the United States: results from the HIV Cost and Services Utilization Study. JAMA 1999;281:2305-15.

24 Max B, Sherer R. Management of the adverse effects of antiretroviral therapy and medication adherence. Clin Infect Dis 2000;30/suppl 2): $596-116$.

25 Report of the NIH panel to define principles of therapy of HIV infection. MMWR Morb Mortal Wkly Rep 1998, Report No 47(RR-5).

26 Sherer $\mathbf{R}$. Adherence and antiretroviral therapy in injection drug users. JAMA 1998;280:567-8

27 Lucas GM, Chaisson RE, Moore RD. Highly active antiretroviral therapy in a large urban clinic: risk factors for virologic failure and adverse drug reactions. Ann Intern Med 1999:131:81-7.

28 Knobel H, Guelar A, Carmona A, et al. Virologic outcome and predictors of virologic failure of highly active antiretroviral therapy containing protease inhibitors. AIDS Patient Care \& Stds containing protease

29 den Brinker M, Wit FW, Wertheim-van Dillen PM, et al. Hepatitis B and $C$ virus co- infection and the risk for hepatotoxicity of highly active antiretroviral therapy in HIV-1 infection. AIDS 2000;14:2895-902.

30 Martinez E, Blanco JL, Arnaiz JA, et al. Hepatotoxicity in HIV-1-infected patients receiving nevirapine-containing antiretroviral therapy. AIDS 2001;15:1261-8.

31 Sulkowski MS, Thomas DL, Chaisson RE, et al. Hepatotoxicity associated with antiretroviral therapy in adults infected with human immunodeficiency virus and the role of hepatitis $C$ or $B$ virus infection. JAMA 2000;283:74-80.

32 Miller KD, Cameron M, Wood LV, et al. Lactic acidosis and hepatic steatosis associated with use of stavudine: report of four cases. Ann Intern Med 2000;133:192-6.

33 Lonergan JT, Behling $\mathrm{C}$, Pfander $\mathrm{H}$, et al. Hyperlactatemia and hepatic abnormalities in 10 human immunodeficiency virus-infected patients receiving nucleoside analogue combination regimens. Clin Infect Dis 2000;31:162-6.

34 Fortgang IS, Belitsos PC, Chaisson RE, et al. Hepatomegaly and steatosis in HIV-infected patients receiving nucleoside analog antiretroviral therapy. Am J Gastroenterol 1995;90:1433-6.

35 ter Hofstede HJ, de Marie S, Foudraine NA, et al. Clinical features and risk factors of lactic acidosis following long-term antiretroviral therapy: 4 risk factors of lactic acidosis following long-term
fatal cases. Int J STD AIDS 2000;1 1:61 1-6.

36 Brinkman K. Editorial response: hyperlactatemia and hepatic steatosis as features of mitochondrial toxicity of nucleoside analogue reverse transcriptase inhibitors. Clin Infect Dis 2000;31:167-9.

37 Durant J, Clevenbergh $P$, Halfon $P$, et al. Drug-resistance genotyping in HIV- 1 therapy: the VIRADAPT randomised controlled trial. Lancet 1999;353:2195-9.

38 Dunne AL, Mitchell FM, Coberly SK, et al. Comparison of genotyping and phenotyping methods for determining susceptibility of HIV- 1 to antiretroviral drugs. AIDS 2001;15:1471-5.

39 Havlir DV, Hellmann NS, Petropoulos CJ, et al. Drug susceptibility in HIV infection after viral rebound in patients receiving indinavir-containing regimens. JAMA 2000;283:229-34.

40 Angel JB, Parato KG, Kumar A, et al. Progressive human immunodeficiency virus-specific immune recovery with prolonged viral suppression. J Infect Dis 2001;183:546-54.

41 Pakker NG, Kroon ED, Roos MT, et al. Immune restoration does not invariably occur following long-term HIV-1 suppression during antiretroviral therapy. INCAS Study Group. AIDS 1999;13:203-12. 
42 Pakker NG, Notermans DW, de Boer RJ, et al. Biphasic kinetics of peripheral blood T cells after triple combination therapy in HIV-1 infection: a composite of redistribution and proliferation. Nat Med 1998;4:208-14.

43 Gorochov G, Neumann AU, Kereveur A, et al. Perturbation of CD4+ and $C D 8+T$-cell repertoires during progression to AIDS and regulation of the CD4+ repertoire during antiviral therapy. Nat Med 1998;4:215-21.

44 Douek DC, Koup RA, McFarland RD, et al. Effect of HIV on thymic function before and after antiretroviral therapy in children. $J$ Infect $D$ is 2000;181:1479-82.

45 Schneider MM, Borleffs JC, Stolk RP, et al. Discontinuation of prophylaxis for Pneumocystis carinii pneumonia in HIV-1-infected patients treated with highly active antiretroviral therapy. Lancet 1999;353:201-3

46 Weverling GJ, Mocroft A, Ledergerber B, et al. Discontinuation of Pneumocystis carinii pneumonia prophylaxis after start of highly active antiretroviral therapy in HIV-1 infection. EuroSIDA Study Group. Lancet 1999;353: 1293-8.
47 Zhang L, Chung C, Hu BS, et al. Genetic characterization of rebounding HIV-l after cessation of highly active antiretroviral therapy. I Clin Invest 2000; 106:839-45

48 Saag MS. The impact of highly active antiretroviral therapy on HIV-specific immune function. AIDS 2001:15(suppl 2):S4-10.

49 Saga MS. The role of immunotherapy in the treatment of HIV AIDS 2001; 15(suppl 2):S1-3.

50 Sperling RS, Shapiro DE, Coombs RW, et al. Maternal viral load, zidovudine treatment, and the risk of transmission of human immunodeficiency virus type 1 from mother to infant. Pediatric AIDS Clinical Trials Group Protocol 076 Study Group. N Engl J Med 1996;335:1621-9.

51 Anonymous. Administration of zidovudine during late pregnancy and delivery to prevent perinatal HIV transmission-Thailand, 1996-1998. MMWR Morb Mortal Wkly Rep 1998;47(8):151-4.

52 Guay LA, Musoke P, Fleming T, et al. Intrapartum and neonatal single-dose nevirapine compared with zidovudine for prevention of mother-to-child transmission of HIV-1 in Kampala, Uganda: HIVNET 012 randomised trial. Lancet 1999;354:795-802

53 Anonymous. Elective caesarean-section versus vaginal delivery in prevention of vertical HIV-1 transmission: a randomised clinical trial. The European Mode of Delivery Collaboration. Lancet 1999;353:1035-9.

\section{Ulcer prophylaxis is unnecessary with short course NSAIDs}

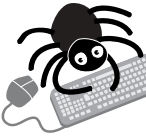

Please visit the Postgraduate Medical Journal website [www. postgradmedj. com] for link to this full article. atients embarking on a short course of non-steroidal anti-inflammatory drugs (NSAIDs) do not need prophylactic treatment against ulcers, even if they are infected with Helicobacter pylori. That is, according to a large randomised, double blind, multicentre clinical trial.

Researchers carefully selected patients with H pylori infection, with no current/ever peptic ulcer, who needed NSAID treatment for musculoskeletal pain. They tested four parallel, prophylactic treatments to compare eradication of $\mathrm{H}$ pylori, or suppression of gastric acid, both, or neither on outcome.

All active treatments significantly reduced ulcers compared with placebo $(0-1.2 \% \vee 5 \%)$, mucosal erosions, and reduced the need for treatment for dyspepsia and were significantly better than placebo on the basis of combined criteria. Treatment to eradicate $H$ pylori or reduce gastric acid was equally effective, but the low proportion of ulcers with placebo suggests that it is unnecessary, the researchers concluded.

From an initial 2264 patients, 832 were randomised to receive one treatment in addition to diclofenac $50 \mathrm{mg}$ twice a day for five weeks. This comprised one week's triple treatment against $H$ pylori (omeprazole $20 \mathrm{mg}$, clarithromycin $500 \mathrm{mg}$, and amoxycillin $\mathrm{lg}$, twice a day) then four weeks' placebo; one week's triple treatment then four weeks' omeprazole (20 mg daily); five weeks' omeprazole (20 mg daily); or five weeks' placebo. Results were analysed for 660 patients.

Conventional NSAIDs are renowned for gastric problems. Whether $H$ pylori reduces or increases ulcers with NSAIDs can be argued either way, and the evidence that its eradication protects against ulcers is controversial.

A Gut 2002;51:329-335 